SCIENTIFIC LETTER

\title{
Impact of combination evidence based medical treatment in patients with acute coronary syndromes in various TIMI risk groups
}

\author{
D Mukherjee, J Fang, E Kline-Rogers, R Otten, K A Eagle
}

Heart 2005;91:381-382. doi: 10.1136/hrt.2004.036459

$\mathrm{T}$ e term acute coronary syndrome (ACS) refers to a spectrum of acute severe cardiac disorders which include unstable angina (UA), non-ST segment elevation myocardial infarction (NSTEMI), and ST segment elevation myocardial infarction (STEMI). Patients presenting with ACS represent a major health problem, accounting for 2.5 million hospitalisations and 500000 deaths annually in the USA alone. ${ }^{1}$

We have previously demonstrated that use of combination evidence based medical treatments including antiplatelet agents, $\beta$ blockers, statins, and angiotensin converting enzyme (ACE) inhibitors is independently and strongly associated with lower six month mortality in patients with ACS. ${ }^{2}$ In the present study we assessed the treatment effect of combination therapy in patients stratified according to their risk of future cardiovascular events according to the thrombolysis in myocardial infarction (TIMI) risk score.

\section{METHODS}

There were 1358 patients who were admitted to, or discharged from, inpatient services at the University of Michigan Medical Center from 1 January 1999 to 11 March 2002 with a diagnosis of ACS. Ninety four patients had documented contraindications to $\beta$ blockers or ACE inhibitors and were excluded from the analysis. The study cohort included the remaining 1264 patients. The study protocol was approved by the institutional review board at the University of Michigan and informed consent was obtained from all patients. Mortality data at six months follow up was obtained for $100 \%$ of the patients based on health system record review and/or telephone call interview.

An appropriateness algorithm for the use of each of the various secondary pharmacologic prevention strategies was created using evidence based clinical practice guidelines from the American College of Cardiology (ACC) and the American Heart Association (AHA), using previously described methodology. ${ }^{2}$ The percentage of patients on appropriate evidence based treatment among those considered eligible was then calculated at hospital discharge. For each patient there were four possible recommended drugs: antiplatelet agents, lipid lowering agents, ACE inhibitors, and $\beta$ blockers. A numerical composite appropriateness score was calculated for each patient on the basis of the number of the drugs used at discharge divided by the number of the drugs indicated.

TIMI risk score is based on the following seven independent risk predictors: (1) age $\geqslant 65$ years; (2) three or more coronary artery disease (CAD) risk factors (family history of $\mathrm{CAD}$, hypertension, hypercholesterolaemia, diabetes, and/or current smoking); ( 3 ) documented CAD ( $\geqslant 50 \%$ stenosis on coronary angiography); (4) aspirin use seven days before hospitalisation; (5) at least two episodes of angina within 24 hours before hospitalisation; (6) ST segment deviation $\geqslant 0.5 \mathrm{~mm}$; (7) elevated cardiac markers. ${ }^{3}$ A full TIMI risk score (points 0-7) was calculated for all patients. The treatment effects at six months were analysed in each risk subgroup and in categories of low (TIMI score 0-3), intermediate (TIMI score 4-5), and high (TIMI score 6-7) risk.

A multivariable logistic regression analysis was performed for six month follow up death and major adverse cardiac event (MACE) in ACS patients with the composite appropriateness variable adjusted for age, sex, positive biomarker, new ST elevation, left ventricular ejection fraction, history of diabetes, renal failure, heart failure, and revascularisation. Both a C index (measure of model discrimination) and Hosmer-Lemeshow test (measure of model calibration) were used to determine the performance of the multivariate models. The number needed to treat (NNT) to prevent an event was calculated based on absolute risk reductions, NNT $=1$ /absolute risk reduction. All analyses were performed using SAS version 8.2 (SAS Institute, Cary, North Carolina, USA).

\section{RESULTS}

The mean (SD) age was 63.7 (13.3) years, and 63\% were male. Co-morbidities included a history of angina in $60.4 \%$, prior myocardial infarction in $42.9 \%$, a history of diabetes mellitus in $30.5 \%$, hypertension in $66.8 \%$, and hyperlipidaemia in $60.6 \%$. Approximately $15 \%$ presented with STEMI, $55 \%$ with NSTEMI, and $30 \%$ with UA. The majority of patients were in either Killip class I or II on presentation.

Two thirds of the patients underwent coronary angiography and approximately $48 \%$ underwent either percutaneous or surgical coronary revascularisation. The use of antiplatelet medications at discharge was approximately $95 \%$, use of $\beta$ blockers was approximately $82 \%$, and among appropriate patients use of ACE inhibitors was $60 \%$ and lipid lowering drugs were prescribed in $84 \%$.

There was a linear increase in mortality and morbidity with increasing severity of TIMI risk categories (fig l). The odds ratio (OR) for death for combination evidence based treatment compared to lack of such treatment in the low risk group was 0.32 (95\% confidence interval (CI) 0.02 to $3.99, \mathrm{p}=0.37)$; in the intermediate risk group it was 0.19 ( $95 \%$ CI 0.03 to $1.15, \mathrm{p}=0.07$ ); and in the high risk group it was 0.14 (95\% CI 0.02 to $0.82, p=0.02$ ) (table 1$)$. The $\mathrm{C}$ indices for the models were $0.82,0.80$, and 0.76 , respectively, suggesting excellent model discrimination. The NNT to

Abbreviations: ACS, acute coronary syndrome; ACC, American College of Cardiology; AHA, American Heart Association; ACE, angiotensin converting enzyme; $C A D$, coronary artery disease; $M A C E$, major adverse cardiac event; NSTEMI, non-ST segment elevation myocardial infarction; NNT, number needed to treat; STEMI, ST segment elevation myocardial infarction; TIMI, thrombolysis in myocardial infarction; UA, unstable angina 


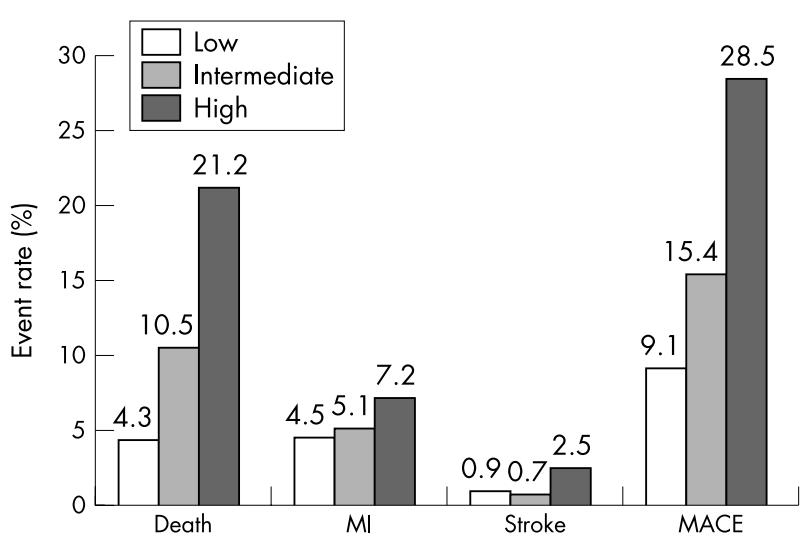

Figure 1 Six month mortality and morbidity in patients with acute coronary syndromes stratified by TIMI risk score into low, intermediate, and high risk groups. MACE, major adverse cardiac events, including death, myocardial infarction, and stroke; Ml, myocardial infarction.

prevent one death was 28 in the low risk group, 18 in the intermediate risk group, and 6 in the high risk group.

\section{DISCUSSION}

Cardiovascular disease remains the leading cause of morbidity and mortality in the USA. ${ }^{1}$ Antiplatelet agents, statins, $\beta$ blockers, and ACE inhibitors are individually very effective in reducing secondary cardiovascular events. However, when prescribed together they may be even more effective and may have incremental and even synergistic benefits in eligible patients. $^{24}$

In this article, we demonstrate that combination evidence based medical treatment was associated with lower six month mortality in patients with ACS with a gradient of benefit across the different TIMI risk groups. The overall mortality reduction in the same patient population has been previously described ${ }^{2}$ and the novel information of this analysis is the gradient of mortality benefit identified according to TIMI risk score. Our data suggest significant synergistic effects of evidence antiplatelet treatment, statins, ACE inhibitors, and $\beta$ blockers, when used together in patients with ACS. A recent hypothetical analysis using Markov modelling of a polypill strategy to simultaneously reduce four cardiovascular risk factors (low density lipoprotein cholesterol, blood pressure, serum homocysteine, and platelet function) demonstrated that combination strategy may reduce cardiovascular disease by more than $80 \%{ }^{5}$

There are several potential limitations of our study. The appropriateness assessment for evidence based treatment was based on ACC/AHA class I guidelines by retrospective review. If patients had previously experienced untoward reactions or contraindications to treatment, which was not documented, this may have been underrepresented with our sampling methodology. This might significantly overestimate
Table 1 Multivariate risk adjusted impact of combination treatment on six month mortality and major adverse cardiac events after acute coronary syndrome

\begin{tabular}{|c|c|c|c|}
\hline & $\begin{array}{l}\text { Low risk group } \\
\text { (TIMI score 0-3) } \\
\text { (n=549) }\end{array}$ & $\begin{array}{l}\text { Intermediate risk group } \\
\text { (TIMI score 4-5) } \\
(n=466 \text { ) }\end{array}$ & $\begin{array}{l}\text { High risk group } \\
\text { (TIMI score 6-7) } \\
\text { ( } n=249 \text { ) }\end{array}$ \\
\hline Death & $\begin{array}{l}0.31(0.02 \text { to } \\
3.99) ; p=0.37\end{array}$ & $\begin{array}{l}0.19(0.03 \text { to } 1.15) \\
p=0.07\end{array}$ & $\begin{array}{l}0.14(0.02 \text { to } \\
0.82) ; p=0.02\end{array}$ \\
\hline MACE & $\begin{array}{l}0.21(0.04 \text { to } \\
0.99) ; p=0.04\end{array}$ & $\begin{array}{l}0.28(0.06 \text { to } 1.19) ; \\
p=0.08\end{array}$ & $\begin{array}{l}0.24(0.04 \text { to } \\
1.22) ; p=0.08\end{array}$ \\
\hline
\end{tabular}

Data are represented as odds ratios with $95 \%$ confidence intervals; data are risk adjusted for age, sex, positive biomarker, new ST elevation, left ventricular ejection fraction, history of diabetes, renal failure, heart failure and revascularisation.

MACE, major adverse cardiac events, including death, myocardial infarction, and stroke.

the potential opportunity to improve secondary preventive measures.

Patients presenting with ACS represent an important high risk cohort, where secondary vascular disease prevention is likely to be particularly effective and cost effective. The benefit of evidence based combination treatment is seen in low, intermediate, and high risk ACS patients with high risk patients deriving the greatest mortality benefit. In other words, the higher the risk, the greater the (absolute and relative) mortality benefit. This is a clinically important message since frequently effective treatments are withheld from patients who would derive the greatest benefit (for example, glycoprotein IIb/IIIa receptor inhibitors in diabetic patients with ACS or early invasive strategy in the elderly).

\section{Authors' affiliations}

D Mukherjee, J Fang, E Kline-Rogers, R Otten, K A Eagle, University of Michigan, Ann Arbor, Michigan, USA

Correspondence to: Dr Debabrata Mukherjee, Division of Cardiology, University of Michigan Health System, University Hospital, TC B1 226, 1500 E Medical Center Drive, Ann Arbor, Michigan 48103-0311, USA; dmukheri@umich.edu

Accepted 24 May 2004

\section{REFERENCES}

1 American Heart Association. 2003 heart and stroke statistical update, Dallas, Texas; 2003.http://www.americanheart.org/downloadable/heart/ 1075102824882HDSStats2004UpdateREV1-23-04.pdf.

2 Mukheriee D, Fang J, Chetcuti S, et al. Impact of combination evidence-based medical therapy on mortality in patients with acute coronary syndromes. Circulation 2004:109:745-9.

3 Antman EM, Cohen M, Bernink PJ, et al. The TIMI risk score for unstable angina/non-ST elevation Ml: a method for prognostication and therapeutic decision making. JAMA 2000;284:835-42.

4 Mukheriee D, Lingam P, Chetcuti S, et al. Missed opportunities to treat atherosclerosis in patients undergoing peripheral vascular interventions: insights from the University of Michigan peripheral vascular disease quality improvement initiative (PVD-Q12). Circulation 2002; 106:1909-12.

5 Wald NJ, Law MR. A strategy to reduce cardiovascular disease by more than $80 \%$. BMJ 2003;326:1419. 